\title{
Impact of Small-for-Gestational Age (SGA) Status on Gentamicin Pharmacokinetics in Neonates
}

The Journal of Clinical Pharmacology 54(I) 39-45

C 2013, The American College of Clinical Pharmacology

DOI: $10.1002 / j c p h .190$

\author{
Mirjana Lulic-Botica, BSc, RPh, BCPS',2, Terri Sheer, PharmD', \\ David Edwards, BScPhm, PharmD, MPH ${ }^{2}$, Ronald L. Thomas, PhD $^{3}$, \\ and Girija Natarajan, $M^{4}$
}

\begin{abstract}
We compared gentamicin pharmacokinetics among neonates born small-for-gestational age (SGA) and appropriate for gestational age (AGA). We further compared gentamicin pharmacokinetics in subgroups of AGA and SGA neonates born preterm and term and treated within and after the initial week of age. Steady state peak and trough serum gentamicin concentrations were used to calculate clearance (Cl), elimination constant (Kel), volume of distribution (Vd), and half-life $\left(t_{1 / 2}\right)$ in infants $(n=236)$ who received $\geq 48$ hours therapy. Statistical analyses (SPSS I 7.0) included chi-square and the nonparametric Mann-Whitney $U$-test. SGA infants treated early $(\leq 7$ days $)(n=29)$ and at postmenstrual ages $\leq 32$ weeks $(n=23)$ had significantly lower median Kel $(0.069 / \mathrm{h}$ vs. $0.08 \mathrm{I} / \mathrm{h}$ and $0.067 / \mathrm{h}$ vs. $0.075 / \mathrm{h})$ and clearance $(0.58 \mathrm{~mL} / \mathrm{kg} / \mathrm{min}$ vs. $0.68 \mathrm{~mL} / \mathrm{kg} / \mathrm{min}$ and $0.46 \mathrm{~mL} / \mathrm{kg} / \mathrm{min} \mathrm{vs}$. $0.65 \mathrm{~mL} / \mathrm{kg} / \mathrm{min})$, compared to those born AGA. There were no significant differences in pharmacokinetic profiles with later therapy or at more mature ages. The prolonged half-life of gentamicin may need to be considered in dosing regimens for preterm SGA infants in the initial week of life.
\end{abstract}

\section{Keywords}

aminoglycoside, gentamicin, pharmacokinetics, small-for gestation

Gentamicin is a commonly used aminoglycoside in neonates, both in empiric and treatment regimens for bacterial pathogens isolated in the Neonatal Intensive Care Unit (NICU). Gentamicin is eliminated unchanged in the urine, almost exclusively by glomerular filtration and has low $(<30 \%)$ protein binding. ${ }^{1-3}$ Gentamicin dosing regimens have included traditional lower doses at more frequent intervals or extended-interval higher dose regimens. ${ }^{1-5}$ The weight-based gentamicin extendedinterval dosing regimens aim to achieve adequate peak concentrations to ensure pathogen killing while avoiding elevated trough concentrations, which may be associated with toxicity. ${ }^{4,6}$ In preterm infants, weight-based dosing regimens are adjusted further for developmental stages or postmenstrual age to account for maturational changes in renal excretion. ${ }^{7,8}$

The effect of small-for-gestational age (SGA) status in neonates on gentamicin pharmacokinetic parameters of clearance and elimination has not been previously examined. SGA status, which may be a result of intrauterine growth restriction (IUGR), may be associated with a decrease in nephron number and renal organ mass, altered tubular function, and impaired glomerular filtration. ${ }^{8-11}$ In limited studies with other medications, the impact of intrauterine growth restriction appears to vary with postnatal and postmenstrual age. ${ }^{7,12-13}$ This may be, in part, due to lower creatinine clearance in the initial 5-10 days of life, specifically in those born preterm. ${ }^{14}$ Further, gestational age, postnatal age, other clinical factors such as hypoxemia have all been shown to affect drug clearance in neonates, with postmenstrual age having the greatest effect on drug clearance. ${ }^{15-17}$ Therefore, the specific aims of this study were to compare gentamicin pharmacokinetic parameters in infants born SGA and those born AGA; and, in subgroups of AGA and SGA infants who were administered the drug early ( $\leq 1$ week of age) and late $(>1$ week) and, finally, in subgroups of AGA and SGA infants whose postmenstrual age at the time of gentamicin administration was $\leq 32$ weeks and $>32$ weeks.

\footnotetext{
'Department of Pharmacy, Hutzel Women's Hospital, Detroit, MI, USA

${ }^{2}$ Department of Pharmacy Practice, Eugene Applebaum College of Pharmacy and Health Sciences, Wayne State University, Detroit, MI, USA

${ }^{3}$ Division of Biostatistics, Children's Hospital of Michigan, Detroit, MI, USA

${ }^{4}$ Division of Neonatology, Children's Hospital of Michigan, Wayne State University, Detroit, MI, USA
}

Submitted for publication 22 March 2013; accepted 17 September 2013.

Corresponding Author: Girija Natarajan, MD, Division of Neonatal-Perinatal Medicine, Children's Hospital of Michigan, 390I Beaubien Blvd, Detroit, MI, USA

Email: gnatara@med.wayne.edu 


\section{Material and Methods}

This was a retrospective chart review of consecutive infants who received gentamicin for $\geq 48$ hours in the NICU at Hutzel Women's Hospital over a 7-year period between 2004 and 2010 and had at least one gentamicin serum concentration measured. The 7-year study period was selected because of availability of electronic searchable pharmacy database; a simplified extendedinterval dosing nomogram was initiated early in the study period and was used for most of the infants. Infants were identified from the electronic database using "gentamicin" and "antibiotics" as search words. The patient list was validated using the pharmacy database. The dosing regimen for early ( $<7$ days of age) onset sepsis was $3 \mathrm{mg} / \mathrm{kg}$ IV q 36 hours for infants $<1,200 \mathrm{~g}, 3 \mathrm{mg} / \mathrm{kg}$ IV q 24 hours for infants $1,200-2,000 \mathrm{~g}$ and $3.5 \mathrm{mg} / \mathrm{kg}$ IV q 24 hours for infants $>2 \mathrm{~kg}$ at birth. For gentamicin use beyond 7 days, doses of $3-4 \mathrm{mg} / \mathrm{kg}$ q 24 hours were administered for infants $<1,200 \mathrm{~g}, 4 \mathrm{mg} / \mathrm{kg}$ q 24 hours for infants who weighed $1,200-2,000 \mathrm{~g}$ and $4-5 \mathrm{mg} / \mathrm{kg} \mathrm{q}$ 24 hours for those who weighed $>2 \mathrm{~kg}$. Gentamicin doses were infused over a 0.5 -hour-period. Permission to access the medical records and electronic databases with waiver of parental consent were obtained from the Wayne State University Investigational Review Board and Detroit Medical Center Research Review.

In our institution, gentamicin concentrations were measured in all infants who were administered the drug for $\geq 48$ hours. Peak (Cpmax) and trough (Cpmin) concentrations of gentamicin were timed with Cpmin being obtained 0.5 hour prior to a dose and Cpmax 0.5 hour following the end of infusion of the dose to allow for distribution of the drug. Clinical pharmacists entered orders for gentamicin levels according to a system protocol and specified the exact time and date for peak and trough collection to ensure that samples were obtained between the third and fourth doses, assumed to be steady state concentrations. Variability in sampling time was minimal. The initial doses for each patient were based on the simplified dosing nomogram. Exact dosing regimens and collection times were noted for the analysis. Table 1 is a description of the distribution of the frequency sampling by dosing regimen. Estimated postdistribution peak concentrations were calculated from two drug concentrations (peak and trough) using a single compartment model and first order SawchukZaske pharmacokinetic equations by the pharmacist. ${ }^{18}$ The gentamicin dose, interval, peak (Cpmax) and trough (Cpmin) concentrations for each patient at assumed steady state were used to calculate clearance of the drug $(\mathrm{Cl})$, volume of distribution $(\mathrm{Vd})$ and the elimination rate constant $\left(\mathrm{kel}^{-1}\right)$. Therapeutic range of Cpmax and Cpmin concentrations were defined as $6-10 \mathrm{mcg} / \mathrm{mL}$ and $<1 \mathrm{mcg} / \mathrm{mL}$, respectively. ${ }^{19}$ Dose
Table I. Distribution of Frequency of Sampling $(n=236)$

\begin{tabular}{lc}
\hline Dose $(\mathrm{mg} / \mathrm{kg} /$ dose $)$ & Frequency \\
\hline $2.5 \mathrm{q} 12$ & 34 \\
$2.5 \mathrm{q} 24$ & 10 \\
$3 \mathrm{q} 24$ & 61 \\
$3 \mathrm{q} 36$ & 32 \\
$3.5 \mathrm{q} 24$ & 49 \\
$3.5 \mathrm{q} 36$ & 30 \\
$4 \mathrm{q} 24$ & 19 \\
$4.5 \mathrm{q} 24$ & 1 \\
Total & 236 \\
\hline
\end{tabular}

adjustments post levels were required for gentamicin peak levels $>10 \mathrm{mcg} / \mathrm{mL}$ or gentamicin trough levels $>1.2 \mathrm{mcg} / \mathrm{mL}$.

Gentamicin serum samples were analyzed using turbidometric inhibition immunoassay technique (Siemens Healthcare Diagnostics). Medical and pharmacy records, and laboratory results were reviewed to obtain neonatal demographic and clinical data. Gestational age was recorded from the medical records from the history and physical and is typically the best obstetric estimate, if available, or a postnatal estimate using Ballard scoring, if unavailable. Infants were classified as AGA (10th90th gender-specific centile) or SGA $(<10$ th genderspecific centile) using the Olsen growth curves. ${ }^{20}$ Postmenstrual age was calculated, as is the convention, as the sum of gestational age (weeks) at birth and the postnatal age (weeks). Assumed steady state gentamicin Cpmax and Cpmin $(\mathrm{mcg} / \mathrm{ml})$ were used to calculate halflife $\left(t_{1 / 2}\right)$ in hours, elimination rate constant $\left(\mathrm{kel}^{-1}\right)$ in hour $^{-1}$, volume of distribution $(\mathrm{Vd})$ in $\mathrm{L} / \mathrm{kg}$ and clearance $(\mathrm{ml} / \mathrm{kg} / \mathrm{min})$. Statistical analysis was performed using SPSS version 17 (SPSS, Inc., Chicago, IL, USA). Descriptive statistics included number (\%), mean (SD) and median (range) values as appropriate. The Shapiro-Wilk test of normality of data revealed a non-normal distribution of pharmacokinetic parameters; therefore all further comparisons of continuous data were performed using the non-parametric Mann-Whitney $U$-test. Comparisons of categorical variables between groups were performed using chi-square test. Significance was taken as a $P$-value $<.05$.

\section{Results}

A total of 236 infants were treated with gentamicin during the study period and underwent therapeutic drug monitoring (TDM). All gentamicin concentrations were obtained after the 3rd dose, which were assumed to be steady state concentrations. A second set of peak and trough Cpmax and Cpmin measurements were obtained in $23(9.7 \%)$ infants and 1 infant had a 3 rd gentamicin peak and trough concentration obtained. 


\section{Clinical Profile}

The mean (SD) gestational age at birth was 30.8 (5.4) weeks and mean (SD) birth weight was $1,641(1,012) \mathrm{g}$. The vast majority [194 (82\%)] of our cohort was born preterm ( $<37$ weeks gestational age). Males comprised $54.7 \%$ of the cohort. Forty-eight (20\%) infants in the study cohort were SGA at birth. The mean (SD) postnatal age at which gentamicin TDM was performed was 13 (19) days. At the time of TDM, serum creatinine levels were $0.5 \mathrm{mg} / \mathrm{dL}$ or less in $75(32 \%)$ infants, between 0.6 and $1 \mathrm{mg} / \mathrm{dL}$ in $133(56 \%)$ infants, between 1.1 and 1.5 in $21(9 \%)$ and above $1.5 \mathrm{mg} / \mathrm{dL}$ in $2(0.8 \%)$ infants; measurements were unavailable in $5(2.2 \%)$ infants. Mean (SD) serum creatinine was $0.7(0.3) \mathrm{mg} / \mathrm{dL}$. An adjustment in gentamicin dosing was required in 54 (23\%) infants, based on initial gentamicin serum concentrations. Bacteria were isolated from the blood in $21(8.9 \%)$ infants; they comprised gram negative bacilli, (12) gram positive cocci (8) and gram positive bacilli. (1) Respiratory endotracheal cultures were positive in 41 (17.4\%) infants for gram negative bacilli, (28) gram positive cocci (5) and multiple organisms. (8) Spinal fluid cultures were performed in 89 (38\%) infants and were negative in all cases. Concomitant vancomycin was administered in 9 and indomethacin in 2 infants.

\section{Effect of SGA Status on Gentamicin TDM}

When infants born SGA $(n=48)$ were compared with those born AGA $(\mathrm{n}=188)$, the median (IQR) birth weights $[752.5(546-1,746) \mathrm{g}$ vs. $1,400(880-2,635) \mathrm{g}$, $P=.0001]$ and weight at the time of TDM [1,265 (610$1,865) \mathrm{g}$ vs. $1572.5 \quad(930-2,599) \mathrm{g}, P=.005]$ were significantly different between groups. The median (IQR) durations of therapy in SGA and AGA infants were identical at $7^{7-10}$ days. All TDM parameters were comparable between groups.

\section{Effect of SGA Status on TDM Parameters With Early} and Late Gentamicin Therapy

We then separately analyzed the effects of SGA status on gentamicin TDM performed within the initial week $(\leq 7$ days) of life. When infants born SGA $(n=29)$ were compared with those born AGA $(n=135)$, the median birth weights and weights at the time of TDM were significantly different (Table 2). In addition, clearance was significantly lower in those born SGA, whereas $T_{1 / 2}$ was significantly longer $(P<.05)$.

Among infants who underwent gentamicin TDM after 7 days of age, there were significant differences between those born SGA $(n=19)$ and those born AGA $(n=53)$ in median birth weights, age at TDM, Cpmin, and serum creatinine (Table 3). At the time of TDM, 39 infants were below the 10th centile for gender-specific postmenstrual age. Of the 19 infants who were SGA at birth, 18 remained below the 10th centile for gender-specific postmenstrual age. The median (IQR) durations of therapy in both groups were 10 (7-14) days.

\section{Effect of SGA Status on TDM Parameters in Preterm Infants With Postmenstrual Age $\leq 32$ Weeks and Those $>32$ Weeks}

Figures 1 and 2 depict mean (SE) clearance and half-lives by postmenstrual age in subgroups of infants born AGA and SGA. Among infants with postmenstrual age $\leq 32$ weeks, those born SGA $(n=23)$ had significantly lower median clearance and prolonged $t_{1 / 2}$, compared to those born AGA $(n=111)$ (Table 4). Among infants with postmenstrual age $>32$ weeks, SGA and AGA infants had comparable pharmacokinetic parameters. The median gestational ages, birth weights, age at TDM and weight at TDM remained significantly different between SGA and AGA subgroups administered gentamicin at $>32$ weeks postmenstrual age.

\section{Discussion}

Among neonates administered a weight-based extendedinterval dosing of gentamicin in the initial week of life and at postmenstrual ages at or below 32 weeks, gentamicin clearance was decreased and half-life was prolonged in

Table 2. Comparison of Median (IQR) Baseline and TDM Parameters Between Groups of SGA $(n=29)$ and AGA $(n=135)$ Infants Who Underwent Gentamicin TDM at $\leq 7$ Days of Life

\begin{tabular}{|c|c|c|c|}
\hline Median (IQR) & SGA $(n=29)$ & $\operatorname{AGA}(n=135)$ & $P$-value by Mann-Whitney U-test \\
\hline Gestational age (weeks) & $30(27-38)$ & $32(27-38)$ & .694 \\
\hline Birth weight (g) & $770(545-2,3 / 2)$ & I,850 (I,030-2,980) & .0001 \\
\hline Gentamicin dose (mg/kg/dose) & $3.1(3.0-3.4)$ & $3.3(2.9-3.5)$ & .082 \\
\hline Age at TDM (days) & $4(3.5-4)$ & $4(3-5)$ & .317 \\
\hline Weight at TDM (g) & $860(535-2,300)$ & I,780 $(920-2,940)$ & .001 \\
\hline Kel $\left(\right.$ hour $\left.^{-1}\right)$ & $0.069(0.050-0.081)$ & $0.081(0.064-0.106)$ & .017 \\
\hline $\mathrm{T}_{1 / 2}$ (hours) & $10(8.5-14.1)$ & $8.6(6.9-10.8)$ & .008 \\
\hline Clearance $(\mathrm{mL} / \mathrm{kg} / \mathrm{min})$ & $0.58(0.4 \mathrm{I}-0.84)$ & $0.68(0.57-0.90)$ & .036 \\
\hline Vd (L/kg) & $0.5(0.4 \mathrm{I}-0.67)$ & $0.5(0.42-0.62)$ & .969 \\
\hline Cpmax (mcg/mL) & $7.7(5.5-8.5)$ & $7.6(6.2-8.6)$ & .645 \\
\hline Cpmin $(\mathrm{mcg} / \mathrm{mL})$ & $1.2(1.1-1.6)$ & I.I (0.8-1.6) & .278 \\
\hline Serum creatinine $(\mathrm{mg} / \mathrm{dL})$ & $0.8(0.5-1.1)$ & $0.8(0.6-0.9)$ & .524 \\
\hline
\end{tabular}


Table 3. Comparison of Median (IQR) Baseline and TDM Parameters Between Groups of SGA $(n=19)$ and AGA $(n=53)$ Infants Who Underwent Gentamicin TDM at $>7$ Days of Life

\begin{tabular}{|c|c|c|c|}
\hline Median (IQR) or $n(\%)$ & $\operatorname{SGA}(n=19)$ & AGA $(n=53)$ & $P$-value \\
\hline Gestational age (weeks) & $28(25-3 I)$ & $27(26-30)$ & 0.847 \\
\hline Birth weight $(\mathrm{g})$ & 750 (545-879) & I,030 (750-I,380) & .004 \\
\hline Gentamicin dose (mg/kg/dose) & $3.1(2.8-3.3)$ & $3.1(2.9-3.5)$ & .599 \\
\hline Age at TDM (days) & $44(2 \mathrm{I}-75)$ & $22(13-42)$ & .021 \\
\hline Weight at TDM $(g)$ & $\mathrm{I}, 420(\mathrm{I}, 020-1,745)$ & $1,280(980-1,703)$ & .848 \\
\hline$<10$ th centile PMA at TDM & $18(95 \%)$ & $21(40 \%)$ & .001 \\
\hline Kel (hour $\left.{ }^{-1}\right)$ & $0.107(0.086-0.124)$ & $0.095(0.08 \mathrm{I}-0.1 \mathrm{II})$ & .195 \\
\hline $\mathrm{T}_{1 / 2}$ (hours) & $6.5(5.6-8.1)$ & $7.3(6.25-8.6)$ & .138 \\
\hline Clearance $(\mathrm{mL} / \mathrm{kg} / \mathrm{min})$ & $0.90(0.77-1.05)$ & $0.80(0.67-0.95)$ & .197 \\
\hline $\operatorname{Vd}(\mathrm{L} / \mathrm{kg})$ & $0.5(0.4-0.6)$ & $0.5(0.4-0.6)$ & .919 \\
\hline Cpmax (mcg/mL) & $7(5.5-8.4)$ & $6.9(5.4-8.05)$ & .964 \\
\hline$C_{p m i n}(\mathrm{mcg} / \mathrm{mL})$ & $0.5(0.3-0.6)$ & $0.7(0.5-0.9)$ & .013 \\
\hline Serum creatinine $(\mathrm{mg} / \mathrm{dL})$ & $0.3(0.3-0.5)$ & $0.5(0.35-0.60)$ & .04 \\
\hline
\end{tabular}

$P$-value by Mann-Whitney U-test.

those born SGA, compared to their AGA counterparts. Beyond a week of age and beyond 32 weeks postmenstrual ages, these differences were no longer apparent. Infants born SGA and administered gentamicin after 1 week of age did have significantly lower trough concentrations. This was probably related to older age and lower serum creatinine in this group, compared to those born AGA.

Clinical pharmacokinetics of aminoglycosides in neonates vary substantially compared to adults due to ongoing developmental changes in drug absorption, distribution, metabolism, and excretion. ${ }^{1}$ The higher total body water and fraction of extracellular water, compared to adults, resulted in higher volume of drug distribution in neonates, with reported values of $0.5-0.7 \mathrm{~L} / \mathrm{kg}$ in premature infants,
$0.2-0.5 \mathrm{~L} / \mathrm{kg}$ in children and $0.2-0.3 \mathrm{~L} / \mathrm{kg}$ in adults respectively. ${ }^{1,22}$ The half-life of gentamicin rapidly changes with postnatal renal maturation, with reported values of 3-11.5 hours in the term neonate $<1$ week of age, 3-5 hours in the older infant and 1.5-3 hours in the adult, respectively. In a recent review of pharmacokinetics in neonates, Pacifici noted variability, with half-lives ranging from 4.9 to 14.6 hours, clearance from 0.53 to $1.72 \mathrm{~mL} / \mathrm{kg}$ / min and volume of distribution ranging from 0.45 to $0.75 \mathrm{~L} / \mathrm{kg} .{ }^{1}$ The author emphasized the need for individualized therapy, especially for premature infants for this reason. ${ }^{1}$ Due to the accelerated maturation of renal tubules, there is an increased clearance of aminoglycosides with increasing gestational ages. ${ }^{23-25}$ Ariano et al. used

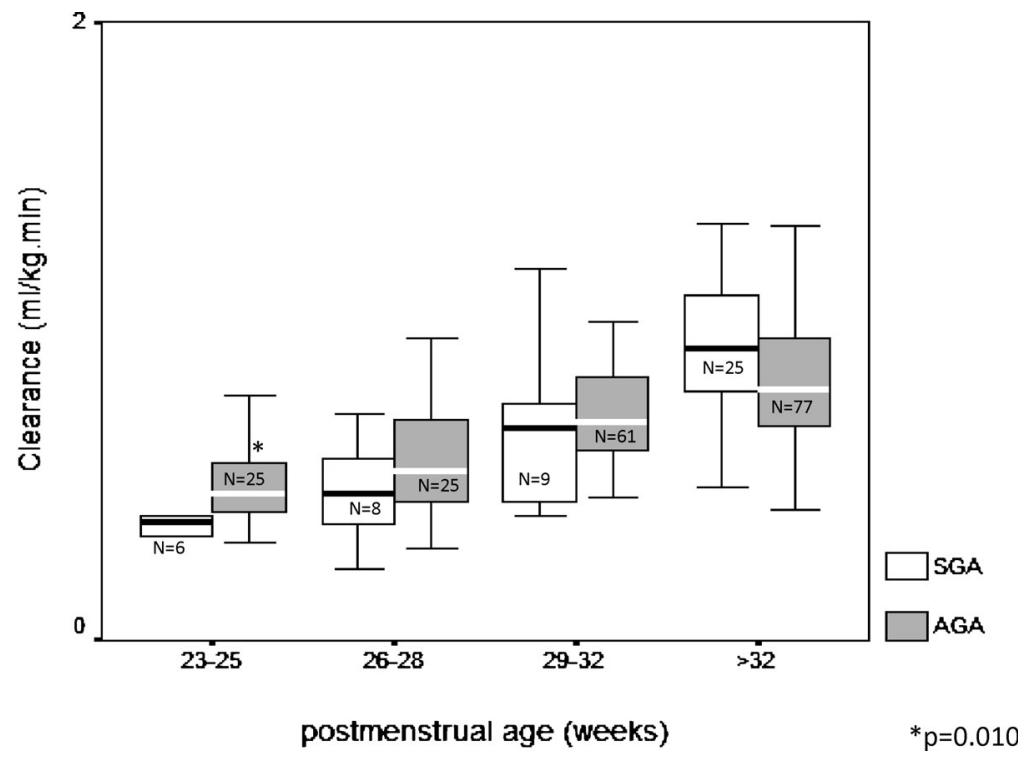

Figure I. Box plot showing median (IQR) clearance $(\mathrm{mL} / \mathrm{kg} / \mathrm{min})$ by postmenstrual age of infants born AGA and SGA. 


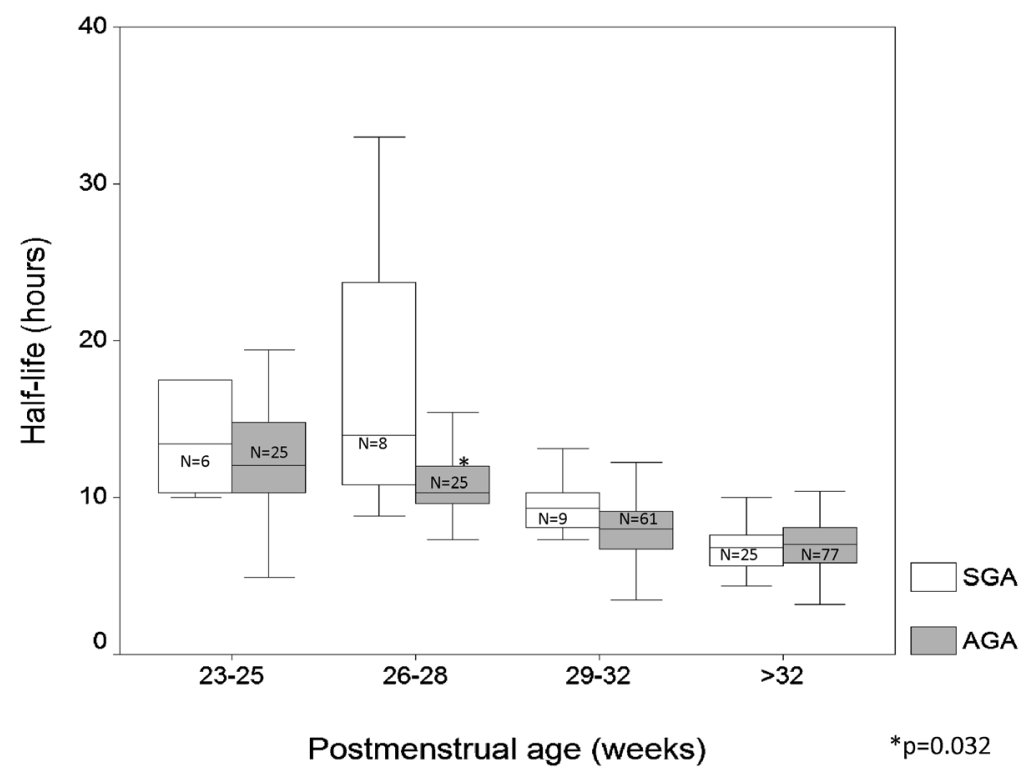

Figure 2. Box plot showing median (IQR) half-life (hours) by postmenstrual age of infants born AGA and SGA.

Bayesian analysis and a dosing nomogram in 58 neonates and found that those born at $\leq 34$ weeks gestation had a weight-normalized apparent volume of gentamicin distribution 1.6 times larger than infants born after 34 weeks' gestation while weight-normalized clearance was $22 \%$ lower. Only $33 \%$ of predicted peak serum gentamicin concentrations were $>6 \mathrm{mg} / \mathrm{L}$ for neonates born at or below 34 weeks' gestation, whereas $90 \%$ were therapeutic in neonates born at older gestations. ${ }^{26}$ Young and Mangum ${ }^{27}$ have previously suggested higher gentamicin doses during the first week of life for infants born at lower gestational ages. In contrast, others have reported that more than gestational age or postnatal age, creatinine clearance, which is related to gestational age, plays an important role in the elimination of gentamicin in premature newborns. ${ }^{2}$ Our data are broadly consistent with previous reported ranges for pharmacokinetic variables.

The effect of varying weights on gentamicin pharmacokinetics in adults has been evaluated in a few studies. Pai et al. $^{28}$ examined gentamicin pharmacokinetics in more than 1,500 adults across the extremes of weight using a variety of body mass descriptors and found that the lean body weight, rather than true body weight or ideal body weight, normalized the volume of distribution across all weight categories and was the best parameter for initial dosing. In another study in adults, dosing weight

Table 4. Comparison of Median (IQR) Baseline and TDM Parameters Between Groups of SGA $(n=23)$ and AGA $(n=I I I)$ Infants Who Underwent Gentamicin TDM at $\leq 32$ Weeks Postmenstrual Age

\begin{tabular}{|c|c|c|c|}
\hline Median (IQR) & SGA $(n=23)$ & $\operatorname{AGA}(n=111)$ & $P$-value \\
\hline Gestational age (weeks) & $27(25-28)$ & $27(25-29)$ & .508 \\
\hline Birth weight $(\mathrm{g})$ & $630(470-750)$ & $1,030(740-1,360)$ & .0001 \\
\hline Gentamicin dose (mg/kg/dose) & $3.1(2.9-3.3)$ & $3.2(2.9-3.5)$ & .176 \\
\hline Age at TDM (days) & $4(4-8)$ & $5(4-13)$ & .286 \\
\hline Weight at TDM (g) & $600(480-860)$ & $\mathrm{I}, 050(750-1,360)$ & .0001 \\
\hline Postmenstrual age at TDM (weeks) & $28(25-30)$ & $29(26-31)$ & .40 \\
\hline Kel $\left(\right.$ hour $\left.^{-1}\right)$ & $0.067(0.046-0.075)$ & $0.075(0.060-0.095)$ & .007 \\
\hline $\mathrm{T}_{1 / 2}$ (hours) & $11.2(9.3-15.1)$ & $9.6(7.6-11.7)$ & .006 \\
\hline Clearance $(\mathrm{mL} / \mathrm{kg} / \mathrm{min})$ & $0.46(0.39-0.69)$ & $0.65(0.53-0.83)$ & .002 \\
\hline $\mathrm{Vd}(\mathrm{L} / \mathrm{kg})$ & $0.5(0.4-0.7)$ & $0.5(0.4-0.7)$ & .825 \\
\hline Cpmax $(\mathrm{mcg} / \mathrm{mL})$ & $8(5.6-8.5)$ & $7(5.8-8.2)$ & .592 \\
\hline Cpmin $(\mathrm{mcg} / \mathrm{mL})$ & $1.2(0.65-1.60)$ & $0.9(0.7-1.4)$ & .318 \\
\hline Serum creatinine $(\mathrm{mg} / \mathrm{dL})$ & $0.9(0.5-1.1)$ & $0.8(0.6-1.0)$ & .243 \\
\hline
\end{tabular}

$P$-value by Mann-Whitney U-test. 
correction factors to give equivalent predicted peak aminoglycoside concentrations with a $2 \mathrm{mg} / \mathrm{kg}$ loading dose were 1.13 times the total body weight for underweight patients. ${ }^{21}$ The effect of SGA status on drug pharmacokinetics in neonates has been examined in a few previous studies. Schreuder et al. ${ }^{16}$ evaluated amikacin clearance in 161 neonates who received amikacin within 24 hours of birth. Birth weight $z$-score and gestational age were correlated with amikacin clearance with partial correlation coefficients of 0.159 and 0.396 , respectively, after correction of other factors. Amikacin clearance was significantly lower in the lowest quartile birth weight $z$-score group of infants, compared to the highest quartile $z$-score $(0.56 \mathrm{~mL} / \mathrm{kg} / \mathrm{min}$ vs. $0.64 \mathrm{~mL} /$ $\mathrm{kg} / \mathrm{min})$. Frattarelli et al. ${ }^{12}$ studied the impact of SGA status on vancomycin pharmacokinetics among 143 infants. Overall Vd, clearance and half-life did not differ between SGA and AGA infants; specific subgroups of SGA infants: $3-4$ weeks old $(0.031 \mathrm{~L} / \mathrm{h}$ vs. $0.088 \mathrm{~L} / \mathrm{h})$ and with a postconceptional age of $27-29$ weeks $(0.021 \mathrm{~L} / \mathrm{h}$ vs. $0.066 \mathrm{~L} / \mathrm{h}$ ) had decreased clearance, compared to infants born AGA. Allegaert et al. ${ }^{15}$ investigated the same research question using population pharmacokinetic studies on preterm neonates within the first month of life for vancomycin (648 drug concentration measures) and amikacin (282 measures). Neonates born small-forgestational age (SGA) were found to have a $16.2 \%$ (coefficient of variation, 12.2\%) reduction in drug clearance from birth up to a postnatal age of 4 weeks. Weight explained $47.3 \%$ of drug clearance; postmenstrual age, $25.2 \%$; co-administration of a nonselective cyclooxygenase inhibitor, 3.5\%; renal function, $7.6 \%$; and SGA, $1.7 \%$. The results of the current and these previous studies are all remarkably consistent; clearance of drugs excreted by kidney is decreased in SGA infants, especially in the early postnatal and postmenstrual weeks of life. In previous studies, postmenstrual age has been shown to be a predictor or major determinant of aminoglycoside clearance, "presumably because it predicts the time course of development of glomerular filtration."15,29

The mechanism of impaired clearance in SGA infants is probably related to low glomerular filtration rate. Intrauterine growth restriction is associated with a reduction in the normalized weight of the kidney, the number of nephrons, the glomerular filtration rate and tubular function. ${ }^{30} \mathrm{~A}$ compensatory hypertrophy with hyperfiltration is also thought to occur in the first months of life. ${ }^{8,9,11}$ Renal blood flow normalized to weight, urine output and fractional excretion of sodium have been shown to be comparable in SGA and AGA animal models, suggesting functional compensation. It is plausible, therefore, that the early reduction in clearance normalizes over time.

Our study has some limitations. Our cohort varied in their gestational ages, postnatal and postmenstrual ages at gentamicin administration and may have had comorbid- ities such as sepsis, and patent ductus arteriosus that may have affected gentamicin clearance. Infants were classified as SGA based on birth weight and may or may not have had IUGR. Our classifications of early and late therapy and the cut-off for postmenstrual ages, although based on previous data and our dosing regimen, were arbitrary. The strengths of our study include the fairly large sample size, our inclusion of all neonates who had gentamicin concentrations measured, our inclusion of several extremely preterm $(43 \% \leq 28$ weeks) infants, a consistent dosing regimen and TDM protocol and the SGA categorization by recent North American Olsen growth curves.

Several studies have shown that dosing of drugs in neonates, especially in preterm neonates, when extrapolated from adult or pediatric studies, often result in variable serum concentrations. In addition, intra and extrauterine growth restriction, although frequent in preterm neonates, have not traditionally been accounted for in neonatal dosing regimens. The current study provides novel insights into the effect of SGA status on gentamicin pharmacokinetics. Our results suggest that, while volume of distribution is unaltered, the prolonged half-life in SGA infants needs to be considered in dosing regimens, especially in the initial week of life and at early postmenstrual ages. We speculate that these alterations may be particularly important in patients with impaired renal function, high-dose or prolonged therapy and with concomitant nephrotoxic medications. Regimens taking the SGA status into consideration may achieve therapeutic drug concentrations more rapidly, reduce the need for dose adjustments and most importantly, may reduce nephrotoxicity, which is related to the renal cortical aminoglycoside concentration. Further pharmacokinetic modeling is required to elucidate the extent of the effect of SGA on gentamicin pharmacokinetics.

\section{Funding}

None.

\section{References}

1. Pacifici GM. Clinical pharmacokinetics of aminoglycosides in the neonate: A review. Eur J Clin Pharmacol. 2009;65:419-427.

2. Garcia B, Barcia E, Perez F, Molina IT. Population pharmacokinetics of gentamicin in premature newborns. J Antimicrob Chemother. 2006;58(2):372-379.

3. Gooding N, Elias-Jones A, Shenoy M. Gentamicin dosing in neonatal patients. Pharm World Sci. 2001;23(5):179-180.

4. Alshaikh B, Dersch-Mills D, Taylor R, Akierman AR, Yusuf K. Extended interval dosing of gentamicin in premature neonates $<28$ week gestation. Acta Paediatr. 2012;101(11):1134-1139.

5. Hagen I, Oymar K. Pharmacological differences between once daily and twice daily gentamicin dosage in newborns with suspected sepsis. Pharm World Sci. 2009;31(1):18-23.

6. Rajchgot P, Prober CG, Soldin S, et al. Aminoglycoside-related nephrotoxicity in the premature newborn. Clin Pharmacol Ther. 1984;35(3):394-401. 
7. Faa G, Gerosa C, Fanni D, et al. Marked interindividual variability in renal maturation of preterm infants: Lesson from autopsy. J Matern Fetal Neonatal Med. 2010, 23(S3):129-133.

8. Chan PY, Morris JM, Leslie GI, Kelly PJ, Gallery ED. The long term effects of prematurity and intrauterine growth restriction on cardiovascular, renal and metabolic function. Int J Pediatr. 2010 280402. doi: 10.1155/2010/280402

9. Hinchliffe SA, Lynch MR, Sargent PH, Howard CV, van Velzen D. The effect of intrauterine growth retardation on the development of renal nephrons. Br J Obstet Gynecol. 1992;99(4):296-301.

10. Manalich R, Reyes L, Herrera M, Melendi C, Fundora I. Relationship between weight at birth and the number and size of renal glomeruli in humans: A histomorphometric study. Kidney Int. 2000;58(2):770-773.

11. Hughson M, Farris AB, Douglas-Denton R, Hoy WE, Bertram JF. Glomerular number and size in autopsy kidneys: The relationship to birth weight. Kidney Int. 2003;63(6):2113-2122.

12. Frattarelli DA, Ergun H, Lulic-Botica M, Lehr VT, Aranda JV. Vancomycin elimination in human infants with intrauterine growth retardation. Pediatr Infect Dis J. 2005;24(11):979-983.

13. Bauer R, Walter B, Zwiener U. Comparison between inulin clearance and endogenous creatinine clearance in newborn normal weight and growth restricted newborn piglets. Exp Toxicol Pathol. 2000;52(4):367-372.

14. Sluncheva B, Dimitrov A, Vakrilova L. Development of renal function in low and extremely low birth weight infants: Correlation with gestational and postnatal age. Akush Ginekol (Sofiia). 2002;41(5):20-26.

15. Allegaert K, Anderson BJ, Van den Anker JN, Vanhaesebrouck S, de Zegher F. Renal drug clearance in preterm neonates: Relation to prenatal growth. Ther Drug Monit. 2007;29(3):284-291.

16. Schreuder MF, Wilhelm AJ, Bokenkamp A, Timmermans SMH, Delemarre-van de Waal HA, van Wijk JAE. Impact of gestational age and birth weight on amikacin clearance on day 1 of life. Clin J Am Soc Nephrol. 2009;4:1774-1778.

17. Myers MG, Roberts RJ, Mirhij NJ. Effects of gestational age, birth weight and hypoxemia on pharmacokinetics of amikacin in serum of infants. Antimicrob Agents Chemother. 1977;11(6):1027-1032.

18. Zaske DE. Aminoglycosides. In: Burton ME, Shaw LM, Schentag JJ, Evans WE, eds. Applied Pharmacokinetics and Pharmacody- namics: Principles of Therapeutic Drug Monitoring. 4th ed. San Francisco, CA: Lippincott Williams and Wilkins; 2005.

19. Touw DJ, Westerman EM, Sprij AJ, Therapeutic drug monitoring of aminoglycosides in neonates. Clin Pharmacokinet. 2009;48(2): 71-88.

20. Olsen IE, Groveman SA, Lawson ML, Clark RH, Zemel BS. New intrauterine growth curves based on United States data. Pediatrics 2010;125:e214-e224.

21. Traynor AM, Nafziger AN, Bertino JS Jr. Aminoglycoside dosing weight correction factors for patients of various body sizes. Antimicrob Agents Chemother. 1995, 39(2):545-548.

22. Shevchuk YM, Taylor DM. Aminoglycoside volume of distribution in pediatric patients. DICP. 1990, 24(3):273-276.

23. Stolk LML, Degraeuwe PLJ, Nieman FHM, et al. Population pharmacokinetics and relationship between demographic and clinical variables and pharmacokinetics of gentamicin in neonates. Ther Drug Monit. 2002;24:527-531.

24. Allegaert K, Anderson BJ. Interindividual variability of aminoglycoside pharmacokinetics in preterm neonates at birth. Eur J Clin Pharamacol. 2006;62:1011-1012.

25. Faura CC, Garcia MR, Horga JF. Changes in gentamicin serum levels and pharmacokinetic parameters in the course of treatment with aminoglycoside. Ther Drug Monit. 1991;13:277-280.

26. Ariano RE, Sitar DS, Davi M, Zelenitsky SA. Bayesian pharmacokinetic analysis of a gentamicin nomogram in neonates: A retrospective study. Curr Ther Res. 2003;64(3):178-188.

27. Young TE, Mangum B, Antibiotics. In: Neofax: A Manual of Drugs Used in Neonatal Care. 20th ed. Montvale, NJ, USA: Thomson Healthcare; 2007: 2-77.

28. Pai MP, Nafziger AN, Bertino JS Jr. Simplified estimation of aminoglycoside pharmacokinetics in underweight and obese adult patients. Antimicrob Agents Chemother. 2011, 55(9):40064011.

29. Sherwin CMT, Svahn S, Van der Linden A, Broadbent RS, Medlicott NJ, Reith DM. Individualized dosing of amikacin in neonates: A pharmacokinetic/pharmacodynamic analysis. Eur J Clin Pharmacol. 2009;65:705-713.

30. Simeoni U, Zetterstrom R. Long-term circulatory and renal consequences of intrauterine growth restriction. Acta Paediatr. 2005;94:819-824. 